\title{
Zolpidem Administration and Risk of Hepatocellular Carcinoma: A Case-Control Study in Taiwan
}

\author{
Shih-Wei Lai ${ }^{1,2}$, Cheng-Li Lin ${ }^{1,3}$ and Kuan-Fu Liao ${ }^{4,5 *}$ \\ ${ }^{1}$ College of Medicine, China Medical University, Taichung, Taiwan, ${ }^{2}$ Department of Family Medicine, China Medical \\ University Hospital, Taichung, Taiwan, ${ }^{3}$ Management Office for Health Data, China Medical University Hospital, Taichung, \\ Taiwan, ${ }^{4}$ College of Medicine, Tzu Chi University, Hualien, Taiwan, ${ }^{5}$ Department of Internal Medicine, Taichung Tzu Chi \\ General Hospital, Taichung, Taiwan
}

OPEN ACCESS

Edited by:

Jean-Paul Deslypere,

Besins Healthcare, Thailand

Reviewed by:

Juan Chen,

Chongqing Medical University, China

Fathi M. Sherif,

University of Tripoli, Libya

${ }^{*}$ Correspondence:

Kuan-Fu Liao

kuanfuliaog@gmail.com

Specialty section: This article was submitted to Pharmaceutical Medicine and Outcomes Research,

a section of the journal

Frontiers in Pharmacology

Received: 21 August 2017 Accepted: 11 October 2017 Published: 23 October 2017

Citation:

Lai S-W, Lin C-L and Liao K-F (2017) Zolpidem Administration and Risk of Hepatocellular Carcinoma: A Case-Control Study in Taiwan.

Front. Pharmacol. 8:767. doi: 10.3389/fphar.2017.00767
Background/Objectives: Previous studies showed that zolpidem use could be associated with increased cancer risk, but the role of zolpidem on hepatocellular carcinoma $(\mathrm{HCC})$ risk remains undetermined. The study purpose was to examine the association between HCC risk and zolpidem use in Taiwan.

Methods: Using the database from the Taiwan National Health Insurance Program, we designed a case-control study which consisted of 77986 subjects aged 20 years or older with newly diagnosed HCC as the case group, and 77986 subjects without HCC as the control group, from 2000 to 2011. Ever use of zolpidem was defined as a subject who had at least a prescription for zolpidem before the index date. Never use was defined as a subject who did not have a prescription for zolpidem before the index date. The association between HCC risk and zolpidem use was determined by the odds ratio (OR) and 95\% confidence interval $(\mathrm{Cl})$ in a multivariable logistic regression model.

Results: After adjustment for confounding factors, the adjusted OR of HCC was 1.05 $(95 \% \mathrm{Cl} 0.97,1.13)$ for subjects with ever use of zolpidem, compared with never use of zolpidem. The adjusted OR of HCC was 1.01 for subjects with increasing cumulative duration of zolpidem use for every 1 year $(95 \% \mathrm{Cl} 0.99,1.03)$, compared with never use of zolpidem.

Conclusion: There is no significant association between HCC risk and zolpidem use. There is no duration-dependent effect of zolpidem use on HCC risk.

Keywords: hepatocellular carcinoma, zolpidem, Taiwan, National Health Insurance Program

\section{INTRODUCTION}

Hepatocellular carcinoma (HCC) ranked the sixth most common cancer (854260 new cases, $4.9 \%$ of the total new cancer cases) and ranked the fourth leading cause of cancer deaths (810000 deaths, $9.3 \%$ of the total cancer deaths) globally in 2015 (Fitzmaurice et al., 2017). HCC ranked the second leading cause of cancer deaths in Taiwan in 2016 (8353 deaths, $17.5 \%$ of the total cancer deaths) (Ministry of Health and Welfare, Taiwan, 2017a).

Zolpidem is a non-benzodiazepine sedative-hypnotic medication commonly used to treat insomnia. To date, no in vitro study examines zolpidem use on cancer risk. Only few epidemiologic studies and review articles showed that zolpidem use could be associated with increased cancer risk 
(Kripke, 2008, 2016; Kripke et al., 2012; Sivertsen et al., 2015). Although drug-induced liver injury has been extensively studied, (Corsini et al., 2012; Hamilton et al., 2016) the case of zolpidem-related liver injury has never been reported and the toxic effect of chronic use of zolpidem on the liver has not been studied. Given that zolpidem is the most frequently prescribed non-benzodiazepine sedative-hypnotic medication in Taiwan, (Su et al., 2002; Hsiao et al., 2013) and HCC is highly prevalent in Taiwan, we make a rational hypothesis that zolpidem use is associated with HCC risk. If the association actually exists, clinicians should be more cautious of zolpidem use. Therefore, we designed a retrospective, population-based, case-control study using the database from the Taiwan National Health Insurance Program to examine whether there is an association between HCC risk and zolpidem use.

\section{MATERIALS AND METHODS}

\section{Data Sources}

Taiwan is an independent country with more than 23 million persons (Chan et al., 2016; Chang and Yu, 2016; Chang et al., 2016; Hsieh et al., 2016; Liang et al., 2017; Liao et al., 2017b; Wen and Yin, 2017; Wu et al., 2017; Yang J.S. et al., 2017; Yang M.D. et al., 2017).

We designed a retrospective, population-based, case-control study using the database from the Taiwan National Health Insurance Program which has covered 99.6\% of 23 million persons living in Taiwan in 2015 (Ministry of Health and Welfare, Taiwan, 2017b). The details of the insurance program can be found in previous studies (Lai et al., 2010, 2011; Chen et al., 2016; Tsai et al., 2016). The study was approved by the Research Ethics Committee of China Medical University and Hospital in Taiwan (CMUH-104-REC2-115).

\section{Inclusion Criteria}

Based on the International Classification of Diseases 9th Revision-Clinical Modification (ICD-9 codes), we defined the cases as subjects aged 20 years or older who were newly diagnosed with HCC (ICD-9 codes 155, 155.0, and 155.2) from 2000 to 2011. We defined the index date for each case as the date of diagnosing HCC. For each case of HCC, we randomly selected one subject without HCC as the control. The HCC cases and the controls were frequency matched by sex, age (within 5 years) and the year of index date.

\section{Exclusion Criteria}

Subjects with other cancers (ICD-9 codes 140-208) before the index date were excluded from the study.

\section{Comorbidities Studied}

The comorbidities before the index date potentially associated with HCC risk were alcohol-related disease, cardiovascular disease, chronic kidney disease, chronic obstructive pulmonary disease, diabetes mellitus, hyperlipidemia, hypertension, as well as chronic liver disease including cirrhosis, hepatitis $\mathrm{B}$ infection, hepatitis C infection, and other chronic hepatitis. All comorbidities were diagnosed based on ICD-9 codes. The diagnosis accuracy of comorbidities was well discussed in previous studies (Lai et al., 2012, 2013; Liao et al., 2016, 2017a; Chu et al., 2017; Lin et al., 2017).

\section{Definition of Medication Exposure}

History of prescriptions for zolpidem and benzodiazepines in Taiwan was included. To minimize a possible confounding effect, subjects whose cumulative duration of zolpidem use was $<12$ months were excluded from the study. Therefore, only subjects whose cumulative duration of zolpidem use was $\geq 12$ months were included. The definition of medication exposure was adapted from previous studies (Cheng et al., 2017; Lai et al., 2017; Liao et al., 2017c,d). Subjects who did not have a prescription for medications studied were classified as never use. Subjects who ever had a prescription for medications studied were classified as ever use.

\section{Statistical Analysis}

The differences in sex, age, zolpidem use, benzodiazepines use, and comorbidities between the HCC cases and the controls were compared by using the Chi-square test for categorized variables and the $t$-test for continuous variables. Variables found to be statistically significant in a univariable logistic regression model were further included in a multivariable logistic regression model. The odds ratio (OR) and 95\% confidence interval were used to estimate the HCC risk associated with zolpidem use. A probability value of $<0.05$ was classified as statistical significance (SAS software version 9.2, SAS Institute Inc., Cary, NC, United States).

\section{RESULTS}

\section{Basic Data of the Study Population}

Totally, 77986 cases with HCC and 77986 controls without HCC were included. Table 1 presents the basic data between the HCC cases and the controls. The HCC cases and the controls had similar proportions of sex and age. The mean ages (standard deviation) were 61.7 (12.4) years in HCC cases and $61.6(12.5)$ years in controls, without statistical significance ( $t$-test, $P=0.06)$. The mean durations of exposure to zolpidem (standard deviation) were 3.06 (2.38) years in HCC cases and 3.03 (3.74) years in controls, without statistical significance $(t$-test, $P=0.71)$.

The HCC cases had higher proportions of zolpidem use (5.39\% vs. $3.32 \%)$, benzodiazepines use (79.9\% vs. $67.4 \%)$, alcohol-related disease ( $14.5 \%$ vs. $3.8 \%)$, chronic kidney disease ( $8.69 \%$ vs. $5.71 \%)$, chronic liver disease ( $84.0 \%$ vs. $13.8 \%)$, chronic obstructive pulmonary disease $(18.6 \%$ vs. $17.9 \%)$, diabetes mellitus (23.9\% vs. $13.8 \%$ ), and hypertension (44.4\% vs. $43.3 \%$ ) 
than the controls, with statistical significance (Chi-square test, $P<0.001$ for all).

\section{Association of Hepatocellular Carcinoma with Zolpidem Use, Benzodiazepines Use, and Comorbidities}

After adjustment for confounding factors, the multivariable logistic regression model presented that the adjusted OR of HCC was 1.05 (95\% CI 0.97, 1.13) for subjects with ever use of zolpidem, compared with those with never use of zolpidem. Other factors significantly associated with increased OR of HCC were benzodiazepines use (adjusted OR 1.50, 95\% CI 1.45, 2.44), alcohol-related disease (adjusted OR 2.31, 95\% CI 2.18, 2.44), chronic kidney disease (adjusted OR 1.21, 95\% CI 1.14, 1.28), chronic liver disease (adjusted OR 34.2, 95\% CI 33.1, 35.2), and diabetes mellitus (adjusted OR 1.93, 95\% CI 1.85, 2.00) (Table 2).

\section{Association of Hepatocellular Carcinoma with Cumulative duration of Zolpidem Use}

We examined whether there was an association between cumulative duration of zolpidem use and HCC risk in Table 3. The sub-analysis presented that the adjusted OR of HCC was 1.01 for subjects with increasing cumulative duration of zolpidem use for every 1 year (95\% CI 0.99, 1.03), compared with those with never use of zolpidem. It seemed that there was no duration-dependent effect of zolpidem use on HCC risk.

\section{DISCUSSION}

In this case-control study, we did not note a significant association between HCC risk and zolpidem use (Table 2). We did not note a duration-dependent effect between HCC risk and zolpidem use (Table 3). It seems that there are inconsistent results about cancer risk and zolpidem use between our study and other previous studies (Kripke, 2008; Kripke et al., 2012; Sivertsen et al., 2015). These previous studies did not adjust for HCC-related risk factors. In our case-control study, risk factors for HCC including alcoholrelated disease, cirrhosis, hepatitis $\mathrm{B}$ infection, hepatitis $\mathrm{C}$ infection, and diabetes mellitus, (Lai et al., 2012) have been included for adjustment. Therefore, the confounding effects by these risk factors might be minimized in our study. That is why there is a difference among these results. The hypothesis that zolpidem use is associated with HCC risk is not convinced at present. It suggests that not all cancers have an association with zolpidem use. More research

TABLE 1 | Basic data between cases with hepatocellular carcinoma and controls.

\begin{tabular}{|c|c|c|c|c|c|}
\hline \multirow[b]{2}{*}{ Variable } & \multicolumn{2}{|c|}{ Controls $N=77986$} & \multicolumn{2}{|c|}{ Cases $N=77986$} & \multirow[b]{2}{*}{$P$-value* } \\
\hline & $n$ & $(\%)$ & $n$ & $(\%)$ & \\
\hline \multicolumn{5}{|l|}{ Sex } & 0.98 \\
\hline Female & 19537 & $(25.1)$ & 19541 & $(25.1)$ & \\
\hline Male & 58449 & $(75.0)$ & 58445 & $(74.9)$ & \\
\hline \multicolumn{5}{|l|}{ Age group (years) } & \multirow[t]{4}{*}{0.99} \\
\hline $20-39$ & 4228 & $(5.4)$ & 4232 & $(5.4)$ & \\
\hline $40-64$ & 39384 & (50.5) & 39383 & $(50.5)$ & \\
\hline $65-84$ & 34374 & $(44.1)$ & 34371 & $(44.1)$ & \\
\hline Age (years), mean \pm standard deviation ${ }^{\dagger}$ & \multicolumn{2}{|c|}{$61.6 \pm 12.5$} & \multicolumn{2}{|c|}{$61.7 \pm 12.4$} & 0.06 \\
\hline Zolpidem use & 2588 & $(3.32)$ & 4207 & $(5.39)$ & $<0.001$ \\
\hline Duration of exposure to zolpidem (years), mean \pm standard deviation ${ }^{\dagger}$ & \multicolumn{2}{|c|}{$3.03 \pm 3.74$} & \multicolumn{2}{|c|}{$3.06 \pm 2.38$} & 0.71 \\
\hline Benzodiazepines use & 52596 & $(67.4)$ & 62321 & $(79.9)$ & $<0.001$ \\
\hline \multicolumn{6}{|l|}{ Comorbidities before index date } \\
\hline Alcohol-related disease & 2961 & $(3.80)$ & 11331 & $(14.5)$ & $<0.001$ \\
\hline Cardiovascular disease & 22404 & $(28.7)$ & 22137 & $(28.4)$ & 0.13 \\
\hline Chronic kidney disease & 4454 & $(5.71)$ & 6778 & $(8.69)$ & $<0.001$ \\
\hline Chronic liver disease & 10769 & $(13.8)$ & 65490 & $(84.0)$ & $<0.001$ \\
\hline Chronic obstructive pulmonary disease & 13940 & $(17.9)$ & 14538 & $(18.6)$ & $<0.001$ \\
\hline Diabetes mellitus & 10735 & $(13.8)$ & 18652 & $(23.9)$ & $<0.001$ \\
\hline Hyperlipidemia & 19966 & (25.6) & 14164 & (18.2) & $<0.001$ \\
\hline Hypertension & 33735 & (43.3) & 34595 & $(44.4)$ & $<0.001$ \\
\hline
\end{tabular}

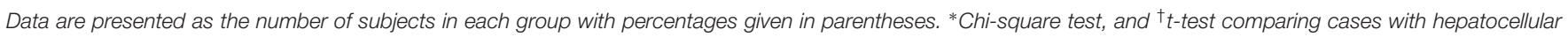
carcinoma and controls. 
TABLE 2 | Odds ratio and 95\% confidence interval of hepatocellular carcinoma associated with zolpidem use, benzodiazepines use, and comorbidities by multivariable logistical regression model.

\begin{tabular}{|c|c|c|c|c|}
\hline \multirow[b]{2}{*}{ Variable } & \multicolumn{2}{|c|}{ Crude } & \multicolumn{2}{|c|}{ Adjusted $^{\dagger}$} \\
\hline & OR & $(95 \% \mathrm{Cl})$ & OR & $(95 \% \mathrm{Cl})$ \\
\hline Sex (male vs. female) & 1.00 & $(0.98,1.02)$ & - & - \\
\hline Age (per one year) & 1.00 & $(0.99,1.00)$ & - & - \\
\hline Zolpidem use (never use as a reference) & 1.66 & $(1.58,1.75)$ & 1.05 & $(0.97,1.13)$ \\
\hline Benzodiazepines use (never use as a reference) & 1.92 & $(1.88,1.97)$ & 1.50 & $(1.45,2.44)$ \\
\hline \multicolumn{5}{|l|}{ Comorbidities before index date (yes vs. no) } \\
\hline Alcohol-related disease & 4.31 & $(4.13,4.49)$ & 2.31 & $(2.18,2.44)$ \\
\hline Cardiovascular disease & 0.98 & $(0.96,1.01)$ & - & - \\
\hline Chronic kidney disease & 1.57 & $(1.51,1.63)$ & 1.21 & $(1.14,1.28)$ \\
\hline Chronic liver disease & 32.7 & $(31.8,33.6)$ & 34.2 & $(33.1,35.2)$ \\
\hline Chronic obstructive pulmonary disease & 1.05 & $(1.03,1.08)$ & 0.85 & $(0.82,0.88)$ \\
\hline Diabetes mellitus & 1.97 & $(1.92,2.02)$ & 1.93 & $(1.85,2.00)$ \\
\hline Hyperlipidemia & 0.65 & $(0.63,0.66)$ & 0.28 & $(0.27,0.29)$ \\
\hline Hypertension & 1.05 & $(1.03,1.07)$ & 0.93 & $(0.90,0.96)$ \\
\hline
\end{tabular}

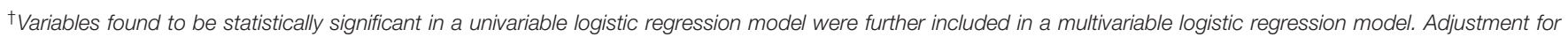

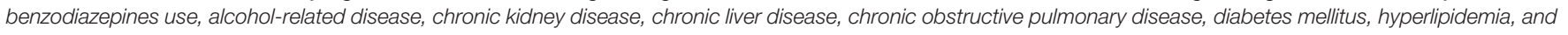
hypertension.

TABLE 3 | The risk of hepatocellular carcinoma associated with cumulative duration of zolpidem use.

\begin{tabular}{|c|c|c|c|c|c|}
\hline Variable & $\begin{array}{c}\text { Case number/control } \\
\text { number }\end{array}$ & Crude OR & $(95 \% \mathrm{Cl})$ & Adjusted OR ${ }^{\dagger}$ & $(95 \% \mathrm{Cl})$ \\
\hline Never use of zolpidem as a reference & $73779 / 75398$ & 1.00 & (reference) & 1.00 & (reference) \\
\hline Cumulative duration of zolpidem use (increase in duration for every 1 year) & $4207 / 2588$ & 1.11 & $(1.10,1.13)$ & 1.01 & $(0.99,1.03)$ \\
\hline
\end{tabular}

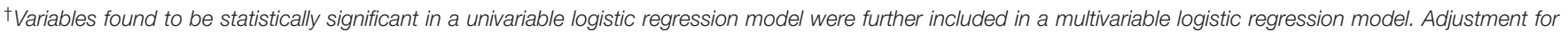

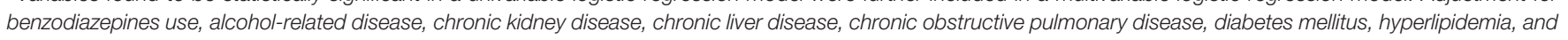
hypertension.

is needed to examine the risk of individual cancer and zolpidem use after adjustment for relevant confounding factors.

Some points need to be discussed. First, an observational case-control study cannot provide a pathophysiological answer for the association between HCC risk and zolpidem use. To date, no in vitro study examines zolpidem use on cancer risk. The case of zolpidem-related liver injury has never been reported and the toxic effect of chronic use of zolpidem on the liver has not been studied. Overall, this is an interesting and novel study in that it attempts to examine the issue for which little evidence is available. Second, HCC is a chronic disease. It is not appropriate to make a link between HCC risk and cumulative duration of zolpidem use $<12$ months. That is why subjects with cumulative duration of zolpidem use $<12$ months were excluded. Only subjects with cumulative duration of zolpidem use $\geq 12$ months were included. Third, due to alpha-fetoprotein not being recorded in the database, we could not include this parameter for analysis. Fourth, we had access to a very useful database from the Taiwan National Health Insurance Program that has contributed to much epidemiological research in the population living in Taiwan. This strength is more convincing to the readers.

\section{CONCLUSION}

There is no significant association between HCC risk and zolpidem use. There is no duration-dependent effect of zolpidem use on HCC risk. Further studies are needed to confirm our findings.

\section{AUTHOR CONTRIBUTIONS}

S-WL planned and conducted this study. He contributed to the conception of the article, initiated the draft of the article, and revised the article. C-LL conducted the data analysis and reviewed the article. K-FL planned and conducted this study. $\mathrm{He}$ participated in the data interpretation and revised the article.

\section{ACKNOWLEDGMENTS}

This study was supported in part by Taiwan Ministry of Health and Welfare Clinical Trial Center (MOHW106-TDU-B-212113004), China Medical University Hospital, Academia Sinica Taiwan Biobank Stroke Biosignature Project (BM10601010036), 
Taiwan Clinical Trial Consortium for Stroke (MOST 106-2321-B039-005), Tseng-Lien Lin Foundation, Taichung, Taiwan, Taiwan Brain Disease Foundation, Taipei, Taiwan, and Katsuzo and Kiyo

\section{REFERENCES}

Chan, C. Y., Lien, C. H., Lee, M. F., and Huang, C. Y. (2016). Quercetin suppresses cellular migration and invasion in human head and neck squamous cell carcinoma (HNSCC). Biomedicine 6:15. doi: 10.7603/s40681-016-0015-3

Chang, L. C., and Yu, Y. L. (2016). Dietary components as epigenetic-regulating agents against cancer. Biomedicine 6:2. doi: 10.7603/s40681-016-0002-8

Chang, W. S., Liu, L. C., Hsiao, C. L., Su, C. H., Wang, H. C., Ji, H. X., et al. (2016). The contributions of the tissue inhibitor of metalloproteinase- 1 genotypes to triple negative breast cancer risk. Biomedicine 6, 23-28. doi: 10.7603/s40681016-0004-6

Chen, H. Y., Lin, C. L., Lai, S. W., and Kao, C. H. (2016). Association of selective serotonin reuptake inhibitor use and acute angle-closure glaucoma. J. Clin. Psychiatry 77, e692-e696. doi: 10.4088/JCP.15m10038

Cheng, K. C., Liao, K. F., Lin, C. L., and Lai, S. W. (2017). Correlation of proton pump inhibitors with pulmonary tuberculosis: a case-control study in Taiwan. Front. Pharmacol. 8:481. doi: 10.3389/fphar.2017.00481

Chu, C. S., Lin, C. C., Peng, C. Y., Chuang, P. H., Su, W. P., Lai, S. W., et al. (2017). Does pyogenic liver abscess increase the risk of delayed-onset primary liver cancer: evidence from a nationwide cohort study. Medicine 96:e7785. doi: 10.1097/MD.0000000000007785

Corsini, A., Ganey, P., Ju, C., Kaplowitz, N., Pessayre, D., Roth, R., et al. (2012). Current challenges and controversies in drug-induced liver injury. Drug Saf. 35, 1099-1117. doi: 10.2165/11632970-000000000-00000

Fitzmaurice, C., Allen, C., Barber, R. M., Barregard, L., Bhutta, Z. A., Brenner, H., et al. (2017). Global, regional, and national cancer incidence, mortality, years of life lost, years lived with disability, and disability-adjusted life-years for 32 cancer groups, 1990 to 2015: a systematic analysis for the global burden of disease study. JAMA Oncol. 3, 524-548. doi: 10.1001/jamaoncol.2016. 5688

Hamilton, L. A., Collins-Yoder, A., and Collins, R. E. (2016). Drug-induced liver injury. AACN Adv. Crit. Care 27, 430-440.

Hsiao, F.-Y., Hsieh, P.-H., and Gau, C.-S. (2013). Ten-year trend in prescriptions of z-hypnotics among the elderly: a nationwide, cross-sectional study in Taiwan. J. Clin. Gerontol. Geriatr. 4, 37-41.

Hsieh, T. C., Wu, Y. C., Sun, S. S., Yen, K. Y., and Kao, C. H. (2016). Treating hepatocellular carcinoma with 90Y-bearing microspheres: a review. Biomedicine 6:19.

Kripke, D. F. (2008). Possibility that certain hypnotics might cause cancer in skin. J. Sleep Res. 17, 245-250. doi: 10.1111/j.1365-2869.2008.00685.x

Kripke, D. F. (2016). Mortality risk of hypnotics: strengths and limits of evidence. Drug Saf. 39, 93-107. doi: 10.1007/s40264-015-0362-0

Kripke, D. F., Langer, R. D., and Kline, L. E. (2012). Hypnotics' association with mortality or cancer: a matched cohort study. BMJ Open 2:e000850. doi: 10.1136/ bmjopen-2012-000850

Lai, S. W., Chen, P. C., Liao, K. F., Muo, C. H., Lin, C. C., and Sung, F. C. (2012). Risk of hepatocellular carcinoma in diabetic patients and risk reduction associated with anti-diabetic therapy: a population-based cohort study. Am. J. Gastroenterol. 107, 46-52. doi: 10.1038/ajg.2011.384

Lai, S. W., Liao, K. F., Lai, H. C., Muo, C. H., Sung, F. C., and Chen, P. C. (2013). Statin use and risk of hepatocellular carcinoma. Eur. J. Epidemiol. 28, 485-492. doi: 10.1007/s10654-013-9806-y

Lai, S. W., Liao, K. F., Liao, C. C., Muo, C. H., Liu, C. S., and Sung, F. C. (2010). Polypharmacy correlates with increased risk for hip fracture in the elderly: a population-based study. Medicine 89, 295-299. doi: 10.1097/MD. 0b013e3181f15efc

Lai, S. W., Lin, C. L., and Liao, K. F. (2017). Use of oral corticosteroids and risk of hip fracture in the elderly in a case-control study. Front. Pharmacol. 8:625. doi: 10.3389/fphar.2017.00625

Lai, S. W., Muo, C. H., Liao, K. F., Sung, F. C., and Chen, P. C. (2011). Risk of acute pancreatitis in type 2 diabetes and risk reduction on anti-diabetic drugs: a
Aoshima Memorial Funds, Japan. These funding agencies did not influence the study design, data collection and analysis, decision to publish, or preparation of the manuscript.

population-based cohort study in Taiwan. Am. J. Gastroenterol. 106, 1697-1704. doi: 10.1038/ajg.2011.155

Liang, Y. C., Hu, J. C., Li, P. Y., Huang, G. J., Kuo, Y. H., and Chao, C. Y. (2017). Torenia concolor Lindley var. formosana Yamazaki extracts improve inflammatory response and lipid accumulation via PPARs activation. Biomedicine 7, 29-36. doi: 10.1051/bmdcn/2017070318

Liao, K. F., Lai, S. W., Lin, C. L., and Chien, S. H. (2016). Appendectomy correlates with increased risk of pyogenic liver abscess: a population-based cohort study in Taiwan. Medicine 95:e4015. doi: 10.1097/MD.0000000000004015

Liao, K. F., Cheng, K. C., Lin, C. L., and Lai, S. W. (2017a). Statin use correlates with reduced risk of pyogenic liver abscess: a population-based case-control study. Basic Clin. Pharmacol. Toxicol. 121, 144-149. doi: 10.1111/bcpt.12777

Liao, K. F., Huang, P. T., Lin, C. C., Lin, C. L., and Lai, S. W. (2017b). Fluvastatin use and risk of acute pancreatitis: a population-based case-control study in Taiwan. Biomedicine 7, 24-28. doi: 10.1051/bmdcn/2017070317

Liao, K.-F., Lin, C.-L., and Lai, S.-W. (2017c). Population-based case-control study assessing the association between statins use and pulmonary tuberculosis in Taiwan. Front. Pharmacol. 8:597. doi: 10.3389/fphar.2017.00597

Liao, K. F., Lin, C. L., and Lai, S. W. (2017d). Nationwide case-control study examining the association between tamoxifen use and Alzheimer's disease in aged women with breast cancer in Taiwan. Front. Pharmacol. 8:612. doi: 10.3389/fphar.2017.00612

Lin, H. F., Liao, K. F., Chang, C. M., Lin, C. L., and Lai, S. W. (2017). Correlation between proton pump inhibitors and risk of pyogenic liver abscess. Eur. J. Clin. Pharmacol. 73, 1019-1025. doi: 10.1007/s00228-017-2256-9

Ministry of Health and Welfare, Taiwan (2017a). 2016 Statistics of Causes of Death. Available at: http://www.mohw.gov.tw/EN/Ministry/Index.aspx

Ministry of Health and Welfare, Taiwan (2017b). 2016 Taiwan Health and Welfare Report. Available at: http://www.mohw.gov.tw

Sivertsen, B., Salo, P., Pentti, J., Kivimaki, M., and Vahtera, J. (2015). Use of sleep medications and risk of cancer: a matched case-control study. Sleep Med. 16, 1552-1555. doi: 10.1016/j.sleep.2015.05.003

Su, T.-P., Chen, T.-J., Hwang, S.-J., Chou, L.-F., Fan, A. P., and Chen, Y.-C. (2002). Utilization of psychotropic drugs in Taiwan: an overview of outpatient sector in 2000. Zhonghua Yi Xue Za Zhi 65, 378-391.

Tsai, T. Y., Lin, C. C., Peng, C. Y., Huang, W. H., Su, W. P., Lai, S. W., et al. (2016). The association between biliary tract inflammation and risk of digestive system cancers: a population-based cohort study. Medicine 95:e4427. doi: 10.1097/MD. 0000000000004427

Wen, Y. J., and Yin, M. C. (2017). The anti-inflammatory and anti-glycative effects of rosmarinic acid in the livers of type 1 diabetic mice. Biomedicine 7, 37-41. doi: 10.1051/bmdcn/2017070319

Wu, M. H., Lee, T. H., Lee, H. P., Li, T. M., Lee, I. T., Shieh, P. C., et al. (2017). Kuei-Lu-Er-Xian-Jiao extract enhances BMP-2 production in osteoblasts. Biomedicine 7:2. doi: 10.1051/bmdcn/2017070102

Yang, J. S., Lu, C. C., Kuo, S. C., Hsu, Y. M., Tsai, S. C., Chen, S. Y., et al. (2017). Autophagy and its link to type II diabetes mellitus. Biomedicine 7:8.

Yang, M.D., Lin, K. C., Lu, M. C., Jeng, L. B., Hsiao, C. L., Yueh, T. C., et al. (2017). Contribution of matrix metalloproteinases-1 genotypes to gastric cancer susceptibility in Taiwan. Biomedicine 7:10. doi: 10.1051/bmdcn/2017070203

Conflict of Interest Statement: The authors declare that the research was conducted in the absence of any commercial or financial relationships that could be construed as a potential conflict of interest.

Copyright (c) 2017 Lai, Lin and Liao. This is an open-access article distributed under the terms of the Creative Commons Attribution License (CC BY). The use, distribution or reproduction in other forums is permitted, provided the original author(s) or licensor are credited and that the original publication in this journal is cited, in accordance with accepted academic practice. No use, distribution or reproduction is permitted which does not comply with these terms. 